\title{
Suspension-Aware Analysis for Hard Real-Time Multiprocessor Scheduling
}

\author{
Cong Liu and James H. Anderson \\ Department of Computer Science, University of North Carolina at Chapel Hill
}

\begin{abstract}
In many real-time systems, tasks may experience suspension delays when accessing external devices. The problem of analyzing task systems with such suspensions on multiprocessors has been relatively unexplored. The commonly used suspension-oblivious approach of treating all suspensions as computation can be quite pessimistic. As an alternative, this paper presents the first suspensionaware hard real-time multiprocessor schedulability analysis for task systems with suspensions, under both global fixed-priority and global EDF scheduling. In experiments presented herein, the proposed schedulability tests proved to be superior to suspension-oblivious tests. Moreover, when applied to ordinary arbitrary-deadline sporadic task systems with no suspensions, the proposed analysis for fixed-priority scheduling improves upon prior analysis.
\end{abstract}

\section{Introduction}

In many embedded systems, suspension delays may occur when tasks block to access shared resources or perform operations on external devices. Such delays can be quite lengthy, in which case schedulability in embedded systems is negatively impacted. For example, delays introduced by disk I/O range from $15 \mu \mathrm{s}$ (for NAND flash) to $15 \mathrm{~ms}$ (for magnetic disks) per read [9]. Delays introduced by accessing devices such as GPUs could range from a few milliseconds to several seconds [7], [15].

It has been shown that precisely analyzing hard real-time (HRT) systems with suspensions is difficult, even for very restricted self-suspending task models on uniprocessors [17]. However, uniprocessor analysis that is correct in a sufficiency sense (although pessimistic) has been proposed (see [16] for an overview). Such analysis can be applied on a per-processor basis to deal with suspensions under partitioning approaches where tasks are statically bound to processors. In contrast, for global scheduling where tasks are scheduled from a single run queue and may migrate across processors, other than the suspension-oblivious approach, which simply integrates suspensions into per-task

Work supported by NSF grants CNS 1239135, CNS 0834270, CNS 0834132, and CNS 1016954; ARO grant W911NF-09-1-0535; AFOSR grant FA9550-09-1-0549; and AFRL grant FA8750-11-1-0033. worst-case-execution-time requirements, no known global HRT schedulability analysis exists for self-suspending task systems. Such approaches are the main focus of this paper.

Unfortunately, unless the number of self-suspending tasks is small and suspension delays are short, the suspension-oblivious approach may cause significant capacity loss. A potentially better alternative is to explicitly consider suspensions in the task model and corresponding schedulability analysis; this is known as suspension-aware analysis. In this paper, we present the first suspensionaware analysis for globally scheduled HRT self-suspending tasks for multiprocessor embedded systems. We focus specifically on two widely used global schedulers: global fixed-priority (GFP) and global EDF (GEDF) scheduling. We analyze these schedulers assuming the scheduled workload is an arbitrary-deadline sporadic self-suspending (SSS) task system (formerly defined in Sec. 2). To enable more general results, we allow soft real-time (SRT) tasks to be supported in addition to HRT ones. Under our definition of SRT, a task can miss a deadline, provided the extent of violation is constrained by a user-specified predefined deadline tardiness threshold. A summary of prior related work and our specific contributions is given next.

Overview of related work. Analysis approaches have been proposed in [13], [14] that can check whether bounded response times can be ensured for any given SSS task system. However, these approaches cannot be used to determine whether hard deadlines or predefined deadline tardiness thresholds (as defined in Sec. II) can be met. An overview of work on scheduling SSS task systems on uniprocessors (which we omit here due to space constraints) can be found in [11], [12], [14]. While (as noted earlier) such work can be applied under partitioning approaches, such approaches suffer from bin-packing-related capacity loss, which limits their usefulness, as we shall see in experiments presented later.

Our contributions. The common suspension-oblivious approach of treating all suspensions as computation for analyzing SSS task systems on multiprocessors (or uniprocessors) is pessimistic. In order to support SSS task systems in a more efficient way, we present in this paper the first suspension-aware global HRT multiprocessor schedulability analysis for general SSS task models. Our specific contributions are as follows. First, we present HRT multipro- 
cessor schedulability tests for arbitrary-deadline SSS task systems under both GFP and GEDF scheduling (note that these tests can also be applied on uniprocessors). Second, we show that our analysis is general enough to also support SRT (with predefined tardiness thresholds) SSS tasks. Third, we demonstrate that, for ordinary arbitrary-deadline task systems with no suspensions, our fixed-priority test has lower time complexity than the best prior test [8]. This is because our test is based on an interval analysis framework tailored to the arbitrary-deadline case, while the test in [8] is based on a framework that encompasses both constrained- and arbitrary-deadline task systems and thus is less exact. Fourth, we present experimental results that show that our suspension-aware analysis is much superior to the prior suspension-oblivious approach. Also, when applied to ordinary arbitrary-deadline task systems with no suspensions, our fixed-priority analysis has better runtime performance than that proposed in [8].

The rest of this paper is organized as follows. In Sec. 2, we present the SSS task model. Then, in Secs. 3 and 4 , we present the aforementioned suspension-aware GFP and GEDF schedulability tests, respectively. In Sec. 5, we experimentally compare them with other methods. In these experiments, our tests exhibited superior performance, typically by a wide margin. We conclude in Sec. 6 .

\section{System Model}

We consider the problem of scheduling an SSS task system $\tau=\left\{\tau_{1}, \ldots, \tau_{n}\right\}$ of $n$ independent SSS tasks on $m \geq 1$ identical processors. Each task is released repeatedly, with each such invocation called a job. Jobs alternate between computation and suspension phases. We assume that each job of any task $\tau_{i}$ executes for at most $e_{i}$ time units (across all of its computation phases) and suspends for at most $s_{i}$ time units (across all of its suspension phases). We place no restrictions on how these phases interleave (a job can even begin or end with a suspension phase). Note that if $s_{i}=0$, then $\tau_{i}$ is an ordinary sporadic task. Associated with each task $\tau_{i}$ are a period $p_{i}$, which specifies the minimum time between two consecutive job releases of $\tau_{i}{ }^{1}$, and a relative deadline $d_{i}$. For any task $\tau_{i}$, we require $e_{i}+s_{i} \leq$ $\min \left(d_{i}, p_{i}\right)$. The $k^{t h}$ job of $\tau_{i}$, denoted $\tau_{i, k}$, is released at time $r_{i, k}$ and has a deadline at time $d_{i, k}=r_{i, k}+d_{i}$. The utilization of a task $\tau_{i}$ is defined as $u_{i}=e_{i} / p_{i}$, and the utilization of the task system $\tau$ as $u_{\text {sum }}=\sum_{\tau_{i} \in \tau} u_{i}$. An SSS task system $\tau$ is said to be an arbitrary-deadline system if, for each $\tau_{i}$, the relation between $d_{i}$ and $p_{i}$ is not constrained (e.g., $d_{i}>p_{i}$ is possible). ${ }^{2}$ In this paper, we consider arbitrary-deadline SSS task systems.

\footnotetext{
${ }^{1}$ In a periodic task system, each task $\tau_{i}$ 's period specifies the exact time between two consecutive job releases of $\tau_{i}$.

${ }^{2} \tau$ is said to be a constrained system if, for each task $\tau_{i} \in \tau, d_{i} \leq p_{i}$.
}

Successive jobs of the same task are required to execute in sequence. If a job $\tau_{i, k}$ completes at time $t$ (that is, its last phase, be it a computation or suspension phase, completes at $t$ ), then its response time is $t-r_{i, k}$ and its tardiness is $\max \left(0, t-d_{i, k}\right)$. A task's response time (tardiness) is the maximum of the response time (tardiness) of any of its jobs. Note that, when a job of a task misses its deadline, the release time of the next job of that task is not altered.

In this paper, we establish HRT schedulability tests for arbitrary-deadline SSS task systems under both GFP (Sec. III) and GEDF (Sec. IV). Under GFP, tasks are assigned fixed priorities; a job has the same priority as the task to which it belongs. Under GEDF, released jobs are prioritized by their deadlines and any ties are broken by task ID (lower IDs are favored).

To enable more general results, for any task $\tau_{i}$, we allow a predefined tardiness threshold to be set, denoted $\lambda_{i}$. (Task $\tau_{i}$ is an ordinary HRT task if $\lambda_{i}=0$.) Throughout the paper, we assume that $e_{i}, s_{i}, d_{i}, p_{i}$, and $\lambda_{i}$ for any task $\tau_{i} \in \tau$ are non-negative integers and all time values are integral. Thus, a job that executes at time point $t$ executes during the entire time interval $[t, t+1)$.

For simplicity, we henceforth assume that each job of any task $\tau_{i}$ executes for exactly $e_{i}$ time units. As shown in [14], any response-time bound derived for an SSS task system by considering only schedules meeting this assumption applies to other schedules as well. (This property was shown in [14] for GEDF, but it applies to GFP as well.) For any job $\tau_{i, k}$, we let $s_{i, k}$ denote its total suspension time, where $s_{i, k} \leq s_{i}$.

Real-time workloads often have both self-suspending tasks and computational tasks (which do not suspend) coexist. To reflect this, we let $\tau^{s}\left(\tau^{e}\right)$ denote the set of selfsuspending (computational) tasks in $\tau$. Also, we let $n_{s}\left(n_{e}\right)$ denote the number of self-suspending (computational) tasks in $\tau$.

\section{GFP Schedulability Test}

In this section, based upon response-time analysis (RTA), we derive a fixed-priority multiprocessor schedulability test for HRT and SRT (i.e., each task $\tau_{i}$ can have a predefined tardiness threshold $\lambda_{i}$ ) arbitrary-deadline SSS task systems.

Under GFP, a task cannot be interfered with by tasks with lower priorities. Assume that tasks are ordered by decreasing priority, i.e., $i<k$ iff $\tau_{i}$ has a higher priority than $\tau_{k}$.

Definition 1. Let $\tau_{l, j}$ be the maximal job of $\tau_{l}$, i.e., $\tau_{l, j}$ either has the largest response time among all jobs of $\tau_{l}$ or it is the first job of $\tau_{l}$ that has a response time exceeding $d_{l}+\lambda_{l}$.

We assume $l>m$ since under GFP, any task $\tau_{i}$ where 


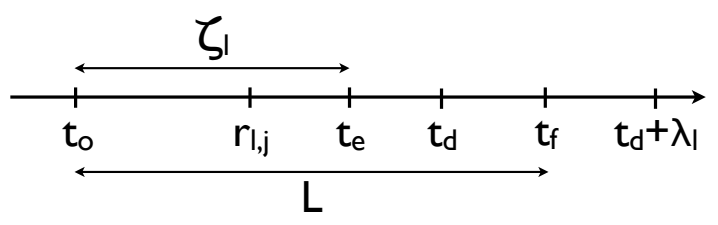

Fig. 1: The maximal job $\tau_{l, j}$ of task $\tau_{l}$ becomes eligible at $t_{e} . t_{o}$ is the earliest time instant before $t_{e}$ such that at any time instant $t \in\left[t_{o}, t_{e}\right)$ all processors are occupied by tasks with equal or higher priority than $\tau_{l, j}$.

$i \leq m$ has a response time bound of $e_{i}+s_{i}$. We further assume that for any task $\tau_{i}$ where $i<l$, its largest response time does not exceed $d_{i}+\lambda_{i}$. Our analysis focuses on the job $\tau_{l, j}$, as defined above. To avoid distracting "boundary cases," we also assume that the schedule being analyzed is prepended with a schedule in which no deadlines are missed that is long enough to ensure that all predecessor jobs referenced in the proof exist (this applies to Sec. IV as well).

Definition 2. A job is said to be completed if it has finished its last phase (be it suspension or computation). $f_{i, k}$ denotes the completion time of job $\tau_{i, k}$. The eligible time of job $\tau_{i, k}$ is defined to be $\max \left(r_{i, k}, f_{i, k-1}\right)$. A task $\tau_{i}$ is active at time $t$ if there exists a job $\tau_{i, k}$ such that $r_{i, k} \leq t<f_{i, k}$.

Definition 3. Let $t_{f}$ denote the completion time of our job of interest $\tau_{l, j}, t_{e}$ denote its eligible time (i.e., $t_{e}=$ $\left.\max \left(r_{l, j}, f_{l, j-1}\right)\right)$, and $t_{d}$ denote its deadline.

As in [2], we extend the analyzed interval from $t_{e}$ to an earlier time instant $t_{o}$ as defined below.

Definition 4. $t_{o}$ denotes the earliest time instant at or before $t_{e}$ such that at any time instant $t \in\left[t_{o}, t_{e}\right)$ all processors are occupied by tasks with equal ${ }^{3}$ or higher priority than $\tau_{l}$, as illustrated in Fig. 1.

For conciseness, let $\tau_{h p} \subseteq \tau$ denote the set of tasks that have equal or higher priority than the analyzed task $\tau_{l}$, and let

$$
L=t_{f}-t_{o}
$$

and

$$
\zeta_{l}=t_{e}-t_{o}
$$

Definition 5. A task $\tau_{i}$ has a carry-in job if there is a job of $\tau_{i}$ that is released before $t_{o}$ that has not completed by $t_{o}$.

Two parameters are important to RTA: the workload and the interference, as defined below.

Workload. The workload of an SSS task $\tau_{i}$ in the interval $\left[t_{o}, t_{f}\right)$ is the amount of computation that $\tau_{i}$ requires to

\footnotetext{
${ }^{3}$ Note that any job $\tau_{l, k}$ of $\tau_{l}$ where $k<j$ may delay $\tau_{l, j}$ from executing and thus can be considered to have higher priority than $\tau_{l, j}$.
}

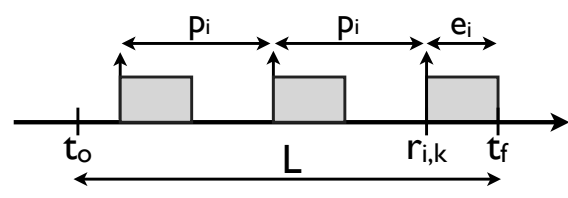

Fig. 2: Computing $\omega^{n c}\left(\tau_{i}, L\right)$.

execute in $\left[t_{o}, t_{f}\right)$. Note that suspensions do not contribute to the workload since they do not occupy any processor. Let $\omega\left(\tau_{i}, L\right)$ denote an upper bound of the workload of each task $\tau_{i} \in \tau_{h p}$ in the interval $\left[t_{o}, t_{f}\right)$ of length $L$. Let $\omega^{n c}\left(\tau_{i}, L\right)$ denote the workload bound if $\tau_{i}$ does not have a carry-in job (see Def. 5), and let $\omega^{c}\left(\tau_{i}, L\right)$ denote the workload bound if $\tau_{i}$ has a carry-in job. $\omega^{n c}\left(\tau_{i}, L\right)$ and $\omega^{c}\left(\tau_{i}, L\right)$ can be computed as shown in the following lemmas.

\section{Lemma 1.}

$$
\omega^{n c}\left(\tau_{i}, L\right)=\left(\left\lfloor\frac{L-e_{i}}{p_{i}}\right\rfloor+1\right) \cdot e_{i}
$$

Proof: Since $\tau_{i}$ does not have a carry-in job, only jobs that are released within $\left[t_{o}, t_{f}\right)$ can contribute to $\omega^{n c}\left(\tau_{i}, L\right)$. The scenario for the worst-case workload to happen is shown in Fig. 2, where job $\tau_{i, k}$, which is the last job of $\tau_{i}$ that is released before $t_{f}$, executes continuously within $\left[r_{i, k}, r_{i, k}+e_{i}\right)$ such that $r_{i, k}+e_{i}=t_{f}$ (according to our task model, each suspension of $\tau_{i, k}$ within $\left[r_{i, k}, t_{f}\right)$ may be of length 0 ), and jobs of $\tau_{i}$ are released periodically. (Note that if $i=l$, then this worst-case scenario still gives a safe upper bound on the workload since in this case $\tau_{l, j}$ could be the job $\tau_{i, k}$.) Besides $\tau_{i, k}$, there are at most $\left\lfloor\frac{L-e_{i}}{p_{i}}\right\rfloor$ jobs of $\tau_{i}$ released within $\left[t_{o}, t_{f}\right)$.

Definition 6. For any interval of length $t$, let $\Delta\left(\tau_{i}, t\right)=$ $\left(\left\lceil\frac{t}{p_{i}}\right\rceil-1\right) \cdot e_{i}+\min \left(e_{i}, t-\left\lceil\frac{t}{p_{i}}\right\rceil \cdot p_{i}+p_{i}\right)$.

$\Delta\left(\tau_{i}, t\right)$ is defined for computing carry-in workloads. Def. 6 improves upon a similar definition for computing carry-in workloads proposed in [10] by deriving a more precise upper bound of the workload.

The following lemma, which computes $\omega^{c}\left(\tau_{i}, L\right)$, is proved similarly to Lemma 1 .

\section{Lemma 2.}

$$
\omega^{c}\left(\tau_{i}, L\right)=\Delta\left(\tau_{i}, L-e_{i}+d_{i}+\lambda_{i}\right)
$$

Proof: The scenario for the worst-case workload to happen is shown in Fig. 3, where job $\tau_{i, k}$, which is the last job of $\tau_{i}$ that is released before $t_{f}$, executes continuously within $\left[r_{i, k}, r_{i, k}+e_{i}\right)$ such that

$$
r_{i, k}+e_{i}=t_{f}
$$

(recall that $\tau_{i, k}$ may suspend for zero time within $\left[r_{i, k}, t_{f}\right)$ ), and jobs are released periodically. (Note that if $i=l$, then 


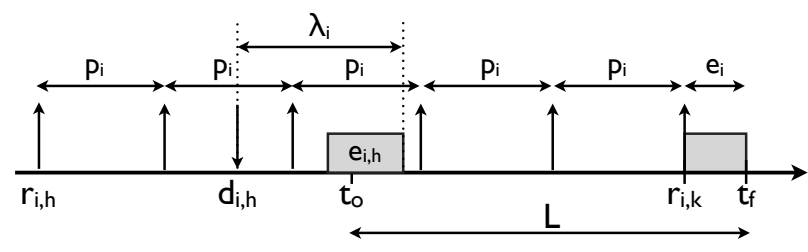

Fig. 3: Computing $\omega^{c}\left(\tau_{i}, L\right)$.

this worst-case scenario still gives a safe upper bound on the workload since $\tau_{l, j}$ could be the job $\tau_{i, k}$.)

Let $\tau_{i, h}$ be the first job such that $r_{i, h}<t_{o}$ and

$$
d_{i, h}+\lambda_{i}>t_{o},
$$

i.e., $\tau_{i, h}$ is the first job of $\tau_{i}$ (potentially tardy) that may execute during $\left[t_{o}, t_{f}\right.$ ) and is released before $t_{o}$ (note that if $\tau_{i, h}$ does not exist, then $\tau_{i}$ would not have a carry-in job). Besides $\tau_{i, k}$ and $\tau_{i, h}$, jobs of $\tau_{i}$ that are released within $\left[r_{i, h}+p_{i}, r_{i, k}\right)$ can contribute to $\omega^{c}\left(\tau_{i}, L\right)$. Let $y$ denote the number of jobs of $\tau_{i}$ released in $\left[r_{i, h}, r_{i, k}\right)$. There are thus $y-1$ jobs of $\tau_{i}$ that are released within $\left[r_{i, h}+p_{i}, r_{i, k}\right)$. Since jobs of $\tau_{i}$ are released periodically, we have

$$
r_{i, k}-r_{i, h}=y \cdot p_{i} .
$$

Moreover, work contributed by $\tau_{i, h}$ cannot exceed the smaller of $e_{i}$ and the length of the interval $\left[t_{o}, d_{i, h}+\lambda_{i}\right)$. The length of the interval $\left[t_{o}, d_{i, h}+\lambda_{i}\right)$ is given by $d_{i, h}+$ $\lambda_{i}-t_{o}=r_{i, h}+d_{i}+\lambda_{i}-t_{o} \stackrel{\{\text { by }}{=}{ }^{(7)\}} r_{i, k}-y \cdot p_{i}+d_{i}+$ $\lambda_{i}-t_{o} \stackrel{\text { by }(1) \stackrel{\text { and }}{=}(5)\}}{=}\left(L-e_{i}+d_{i}+\lambda_{i}\right)-y \cdot p_{i}$. Thus, the work contributed by $\tau_{i, h}$ is given by $\min \left(e_{i},\left(L-e_{i}+\right.\right.$ $\left.\left.d_{i}+\lambda_{i}\right)-y \cdot p_{i}\right)$.

By summing the contributions of $\tau_{i, h}, \tau_{i, k}$, and jobs of $\tau_{i}$ that are released within $\left[r_{i, h}+p_{i}, r_{i, k}\right)$, we have

$$
\begin{aligned}
& \omega^{c}\left(\tau_{i}, L\right) \\
= & \min \left(e_{i},\left(L-e_{i}+d_{i}+\lambda_{i}\right)-y \cdot p_{i}\right) \\
& +e_{i}+(y-1) \cdot e_{i} \\
= & \min \left(e_{i},\left(L-e_{i}+d_{i}+\lambda_{i}\right)-y \cdot p_{i}\right) \\
& +y \cdot e_{i}
\end{aligned}
$$

To find $y$, by (7), we have $y=\frac{r_{i, k}-r_{i, h}}{p_{i}}=\frac{r_{i, k}-d_{i, h}+d_{i}}{p_{i}}$ $\{$ by $(6)\} \frac{r_{i, k}-t_{o}+\lambda_{i}+d_{i}}{p_{i}}\left\{\right.$ by (1) and(5)\} $\frac{L-e_{i}+\lambda_{i}+d_{i}}{=}$. For conciseness, let $\sigma=L-e_{i}+\lambda_{i}+d_{i}$. Thus, $y<\frac{\sigma}{p_{i}}$ holds. If $\sigma \bmod p_{i}=0$, then $y \leq \frac{\sigma}{p_{i}}-1=\left\lceil\frac{\sigma}{p_{i}}\right\rceil-1$, otherwise, $y \leq\left\lfloor\frac{\sigma}{p_{i}}\right\rfloor=\left\lceil\frac{\sigma}{p_{i}}\right\rceil-1$. Thus, a general expression for $y$ can be given by $y \leq\left\lceil\frac{\sigma}{p_{i}}\right\rceil-1$.

By (8), the maximum value for $\omega^{c}\left(\tau_{i}, L\right)$ can be obtained when $y=\left\lceil\frac{\sigma}{p_{i}}\right\rceil-1$. Setting this expression for $y$ into (8), we get $\omega^{c}\left(\tau_{i}, L\right)=\min \left(e_{i}, \sigma-\left\lceil\frac{\sigma}{p_{i}}\right\rceil \cdot p_{i}+\right.$

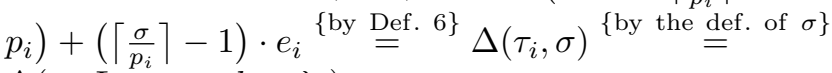
$\Delta\left(\tau_{i}, L-e_{i}+d_{i}+\lambda_{i}\right)$.
It is important to point out that neither $\omega^{n c}\left(\tau_{i}, L\right)$ nor $\omega^{c}\left(\tau_{i}, L\right)$ depends on $\zeta_{l}$ (as defined in (2)). For any given interval $\left[t_{o}, t_{f}\right)$ of length $L$, we get the same result of $\omega^{n c}\left(\tau_{i}, L\right)$ and $\omega^{c}\left(\tau_{i}, L\right)$, regardless of the value of $\zeta_{l}$. This observation enables us to greatly reduce the time complexity to derive the response time bound, as shown later.

Interference. The interference $I_{l}\left(\tau_{i}, L\right)$ of a specific task $\tau_{i}$ on $\tau_{l}$ over $\left[t_{o}, t_{f}\right)$ is the part of the workload of $\tau_{i}$ that has higher priority than $\tau_{l, j}$ and can delay $\tau_{l, j}$ from executing its computation phases. Note that if $i \neq l$, then $\tau_{i}$ cannot interfere with $\tau_{l}$ while $\tau_{i}$ or $\tau_{l}$ is suspending. If $i=l$, then suspensions of job $\tau_{l, k}$ where $k<j$, may delay $\tau_{l, j}$ from executing. However, by Def. 4 , all processors are occupied by tasks with equal or higher priority than $\tau_{l}$ at any time instant $t \in\left[t_{o}, t_{e}\right)$. Thus, whenever suspensions of any such job $\tau_{l, k}$ delay $\tau_{l, j}$ from executing within $\left[t_{o}, t_{e}\right)$, such suspensions must be overlapped with computation from some other task with higher priority than $\tau_{l}$. Therefore, it suffices for us to compute the interference using workload as derived in (3) and (4). (Intuitively, this portion of the schedule, i.e., the schedule within $\left[t_{o}, t_{e}\right)$, would be the same even if $\tau_{l}$ did not suspend, since $\tau_{l}$ has the lowest priority among the tasks being considered.)

As we did for the workload, we also define two expressions for $I_{l}\left(\tau_{i}, L\right)$. We use $I_{l}^{n c}\left(\tau_{i}, L\right)$ to denote a bound on the interference of $\tau_{i}$ to $\tau_{l}$ during $\left[t_{o}, t_{f}\right)$ if $\tau_{i}$ does not have a carry-in job, and use $I_{l}^{c}\left(\tau_{i}, L\right)$ if $\tau_{i}$ has a carry-in job.

By the definitions of workload and interference, within $\left[t_{o}, t_{f}\right)$, if $i \neq l$, then task $\tau_{i}$ cannot interfere with $\tau_{l}$ by more than $\tau_{i}$ 's workload in this interval. Thus, we have $I_{l}^{n c}\left(\tau_{i}, L\right) \leq \omega^{n c}\left(\tau_{i}, L\right)$ and $I_{l}^{c}\left(\tau_{i}, L\right) \leq \omega^{c}\left(\tau_{i}, L\right)$. The other case is $i=l$. In this case, since $\tau_{l, j}$ cannot interfere with itself, we have $I_{l}^{n c}\left(\tau_{i}, L\right) \leq \omega^{n c}\left(\tau_{l}, L\right)-e_{l}$ and $I_{l}^{c}\left(\tau_{i}, L\right) \leq \omega^{c}\left(\tau_{l}, L\right)-e_{l}$. Moreover, because $\tau_{i}$ cannot interfere with $\tau_{l}$ while $\tau_{l, j}$ is executing and suspending for a total of $e_{l}+s_{l, j}$ time units in $\left[t_{o}, t_{f}\right), I_{l}\left(\tau_{i}, L\right)$ cannot exceed $L-e_{l}-s_{l, j}$. Therefore, we have ${ }^{4}$

$$
\begin{aligned}
& I_{l}^{n c}\left(\tau_{i}, L\right)= \\
& \begin{cases}\min \left(\omega^{n c}\left(\tau_{i}, L\right), L-e_{l}-s_{l, j}+1\right), & \text { if } i \neq l \\
\min \left(\omega^{n c}\left(\tau_{l}, L\right)-e_{l}, L-e_{l}-s_{l, j}+1\right), & \text { if } i=l\end{cases}
\end{aligned}
$$

and

$$
\begin{aligned}
& I_{l}^{c}\left(\tau_{i}, L\right)= \\
& \begin{cases}\min \left(\omega^{c}\left(\tau_{i}, L\right), L-e_{l}-s_{l, j}+1\right), & \text { if } i \neq l \\
\min \left(\omega^{c}\left(\tau_{l}, L\right)-e_{l}, L-e_{l}-s_{l, j}+1\right), & \text { if } i=l .\end{cases}
\end{aligned}
$$

${ }^{4}$ The upper bounds of $I_{l}^{n c}\left(\tau_{i}, L\right)$ and $I_{l}^{c}\left(\tau_{i}, L\right)$ (as shown next) are set to be $L-e_{l}-s_{l, j}+1$ instead of $L-e_{l}-s_{l, j}$ in order to guarantee that the response time bound we get from the schedulability test presented later is valid. A formal explanation of this issue can be found in [5]. 
Now we define the total interference bound on $\tau_{l}$ within any interval $\left[t_{o}, t_{o}+Z\right)$ of arbitrary length $Z$, denoted $\Omega_{l}(Z)$, which is given by $\sum_{\tau_{i} \in \tau_{h p}} I_{l}\left(\tau_{i}, Z\right)$. The total interference bound on $\tau_{l}$ within the interval $\left[t_{o}, t_{f}\right)$ is thus given by $\Omega_{l}(L)$.

Upper-bounding $\Omega_{l}(L)$. By Def. 4 , either $t_{o}=0$, in which case no task has a carry-in job, or some processor is idle in $\left[t_{o}-1, t_{o}\right)$, in which at most $m-1$ computational tasks are active at $t_{o}-1$. Thus, at most $\min \left(m-1, n_{h p}^{e}\right)$ computational tasks in $\tau_{h p}$ have carry-in jobs, where $n_{h p}^{e}$ denotes the number of computational tasks in $\tau_{h p}$. Due to suspensions, however, all self-suspending tasks in $\tau_{h p}$ may have carry-in jobs that suspend at $t_{o}$. Let $\tau_{h p}^{s}$ denote the set of self-suspending tasks in $\tau_{h p}$. Thus, self-suspending tasks can contribute at most $\sum_{\tau_{i} \in \tau_{h p}^{s}} \max \left(I_{l}^{c}\left(\tau_{i}, L\right), I_{l}^{n c}\left(\tau_{i}, L\right)\right)$ work to $\Omega_{l}(L)$. Let $\tau_{h p}^{e}$ denote the set of computational tasks in $\tau_{h p}$ and $\beta_{\tau_{i} \in \tau_{h p}^{e}}^{\min \left(m-1, n_{h p}^{e}\right)}$ denote the $\min (m-$ $\left.1, n_{h p}^{e}\right)$ greatest values of $\max \left(0, I_{l}^{c}\left(\tau_{i}, L\right)-I_{l}^{n c}\left(\tau_{i}, L\right)\right)$ for any computational task $\tau_{i} \in \tau_{h p}^{e}$. Then computational tasks can contribute at most $\sum_{\tau_{i} \in \tau_{h p}^{e}} I_{l}^{n c}\left(\tau_{i}, L\right)+\beta_{\tau_{i} \in \tau_{h p}^{e}}^{\min \left(m-1, n_{h p}^{e}\right)}$ work to $\Omega_{l}(L)$. Therefore, by summing up the work contributed by both self-suspending tasks and computational tasks, we can bound $\Omega_{l}(L)$ by

$$
\begin{aligned}
\Omega_{l}(L)= & \sum_{\tau_{i} \in \tau_{h p}^{s}} \max \left(I_{l}^{c}\left(\tau_{i}, L\right), I_{l}^{n c}\left(\tau_{i}, L\right)\right) \\
& +\sum_{\tau_{i} \in \tau_{h p}^{e}} I_{l}^{n c}\left(\tau_{i}, L\right)+\beta_{\tau_{i} \in \tau_{h p}^{e}}^{\min \left(m-1, n_{h p}^{e}\right)}
\end{aligned}
$$

The time complexity for computing $\sum_{\tau_{i} \in \tau_{h p}^{s}} \max \left(I_{l}^{c}\left(\tau_{i}, L\right), \quad I_{l}^{n c}\left(\tau_{i}, L\right)\right) \quad$ and $\sum_{\tau_{i} \in \tau_{h p}^{e}} I_{l}^{n c}\left(\tau_{i}, L\right)$ is $O(n)$. Also, as noted in [2], by using a linear-time selection technique from [6], the time complexity for computing $\beta_{\tau_{i} \in \tau_{h p}^{e}}^{\min \left(m-1, n_{h p}^{e}\right)}$ is $O(n)$. Thus, the time complexity to upper-bound $\Omega_{l}(L)$ as above is $O(n)$.

Schedulability test. We now derive an upper bound on the response time of task $\tau_{l}$ in an SSS task system $\tau$ scheduled using fixed priorities, as stated in Theorem 1. Before stating the theorem, we first present two lemmas, which are used to prove the theorem. Lemma 3 is intuitive since it states that the total interference of tasks with equal or higher priority than $\tau_{l}$ must be large enough to prevent $\tau_{l, j}$ from being finished at $t_{o}+H$ if $t_{o}+H<t_{f}$ holds (recall that $t_{f}$ is defined to be the completion time of $\tau_{l, j}$ ).

Lemma 3. For job $\tau_{l, j}$ and any interval $\left[t_{o}, t_{o}+H\right)$ of length $H$, if $H<t_{f}-t_{o}$, then

$$
\left\lfloor\frac{\Omega_{l}(H)}{m}\right\rfloor>H-e_{l}-s_{l, j} .
$$

Proof: $\Omega_{l}(H)$ denotes the total interference bound on $\tau_{l}$ within the interval $\left[t_{o}, t_{o}+H\right)$.

If $t_{e} \geq t_{o}+H$, then by Def. 4 , all processors must be occupied by tasks in $\tau_{h p}$ during the interval $\left[t_{o}, t_{o}+H\right)$, which implies that tasks in $\tau_{h p}$ generate a total workload of at least $m \cdot H$ within $\left[t_{o}, t_{o}+H\right)$ that can interfere with $\tau_{l}$. Thus, (12) holds since $\Omega_{l}(H) \geq m \cdot H \geq m \cdot\left(H-e_{l}-\right.$ $\left.s_{l, j}+1\right)$.

The other possibility is $t_{e}<t_{o}+H$. In this case, given (from the statement of the lemma) that $H<t_{f}-t_{o}$, job $\tau_{l, j}$ is not yet completed at time $t_{o}+H$. Thus, only at strictly fewer than $e_{l}+s_{l, j}$ time points within the interval $\left[t_{o}, t_{o}+H\right)$ was $\tau_{l, j}$ able to execute its computation and suspension phases (for otherwise it would have completed by $\left.t_{o}+H\right)$. In order for $\tau_{l, j}$ to execute its computation and suspension phases for strictly fewer than $e_{l}+s_{l, j}$ time points within $\left[t_{o}, t_{o}+H\right)$, tasks in $\tau_{h p}$ must generate a total workload of at least $m \cdot\left(H-e_{l}-s_{l, j}+1\right)$ within $\left[t_{o}, t_{o}+H\right)$ that can interfere with $\tau_{l}$. Thus, $\Omega_{l}(H) \geq$ $m \cdot\left(H-e_{l}-s_{l, j}+1\right)$ holds.

Lemma 4. $t_{e}-r_{l, j} \leq \kappa_{l}$, where $\kappa_{l}=\lambda_{l}-p_{l}+d_{l}$ if $\lambda_{l}>p_{l}-d_{l}$, and $\kappa_{l}=0$, otherwise.

Proof: By Def. 1, we have

$$
f_{l, j-1} \leq d_{l, j-1}+\lambda_{l}
$$

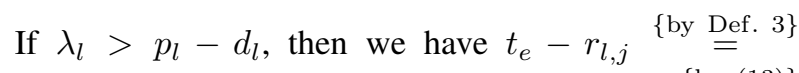
$\max \left(r_{l, j}, f_{l, j-1}\right)-r_{l, j}=\max \left(0, f_{l, j-1}-r_{l, j}\right) \stackrel{\{\text { by }}{\leq}$ $\max \left(0, d_{l, j-1}+\lambda_{l}-r_{l, j}\right)=\max \left(0, r_{l, j-1}+d_{l}+\lambda_{l}-r_{l, j}\right) \leq$ $\max \left(0, d_{l}+\lambda_{l}-p_{l}\right)=\lambda_{l}-p_{l}+d_{l}$.

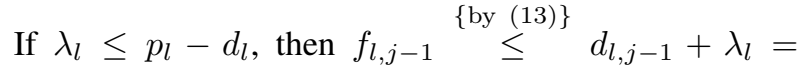
$r_{l, j-1}+d_{l}+\lambda_{l} \leq r_{l, j}-p_{l}+d_{l}+\lambda_{l} \leq r_{l, j}$, which implies that job $\tau_{l, j-1}$ completes by $r_{l, j}$. Thus, by Def. 3 , we have $t_{e}-r_{l, j}=0$.

Theorem 1. Let $\psi_{l}$ be the set of minimum solutions of (14) for $L$ below for each value of $s_{l, j} \in\left\{0,1,2, \ldots, s_{l}\right\}$ by performing a fixed-point iteration on the RHS of (14) starting with $L=e_{l}+s_{l, j}$ :

$$
L=\left\lfloor\frac{\Omega_{l}(L)}{m}\right\rfloor+e_{l}+s_{l, j} .
$$

Then $\psi_{l}^{\text {max }}+\kappa_{l}$ upper-bounds $\tau_{l}$ 's response time, where $\psi_{l}^{\max }$ is the maximum value in $\psi_{l}$.

Proof: We first prove by contradiction that $\psi_{l}^{\max }+$ $\kappa_{l}-\zeta_{l}$ is an upper bound of $\tau_{l}$ 's response time. Assume that the actual worst-case response time of $\tau_{l}$ is given by $R$, where

$$
R>\psi_{l}^{\max }+\kappa_{l}-\zeta_{l}
$$


By Defs. 1 and 3, we have

$$
R=t_{f}-r_{l, j} \text {. }
$$

Thus, we have $\left.\psi_{l}^{\max } \stackrel{\text { by }}{<}{ }^{<} R+\zeta_{l}-\kappa_{l} \stackrel{\text { by }}{\stackrel{(16)}{=}}\right\}$ $t_{f}-r_{l, j}+\zeta_{l}-\kappa_{l} \stackrel{\text { by }(2)\}}{=} t_{f}-t_{o}+t_{e}-r_{l, j}-\kappa_{l} \quad$ by Lemma 4\} $t_{f}-t_{o}+\kappa_{l}-\kappa_{l}=t_{f}-t_{o}$. Hence, by Lemma $\overline{3}$, (12) holds with $H=\psi_{l}^{\max }$, which contradicts the assumption of $\psi_{l}^{\max }$ being a solution of (14). Therefore, $\psi_{l}^{\max }+\kappa_{l}-\zeta_{l}$ is an upper bound of $\tau_{l}$ 's response time.

By (2) and Def. 4, $\zeta_{l} \geq 0$ holds. Moreover, by (3) and (4)-(11), $\Omega_{l}(L)$ is independent of $\zeta_{l}$, which implies that $\psi_{l}^{\max }$ is independent of $\zeta_{l}$. Thus, the maximum value for the term $\psi_{l}^{\max }+\kappa_{l}-\zeta_{l}$, which is given by $\psi_{l}^{\max }+\kappa_{l}$ when setting $\zeta_{l}=0$, is an upper bound of $\tau_{l}$ 's response time.

Note that (14) depends on $s_{l, j}$. Thus, it is necessary to test each possible value of $s_{l, j} \in\left\{0,1,2, \ldots, s_{l}\right\}$ to find a corresponding minimum solution of (14). By the definition of $\psi_{l}^{\max }, \psi_{l}^{\max }$ can then be safely used to upper-bound $\tau_{l}$ 's response time. Moreover, for every task $\tau_{i} \in \tau, \psi_{i}^{\max } \leq$ $d_{i}+\lambda_{i}-\kappa_{i}$ must hold in order for $\tau$ to be schedulable; otherwise, some jobs of $\tau_{i}$ may have missed their deadlines by more than the corresponding tardiness thresholds. The following corollary immediately follows.

Corollary 1. Task system $\tau$ is GFP-schedulable upon $m$ processors if, by repeating the iteration stated in Theorem 1 for all tasks $\tau_{i} \in \tau, \psi_{i}^{\max } \leq d_{i}+\lambda_{i}-\kappa_{i}$ holds.

Comparing with [8]. In [8], an RTA technique, which we refer to as "GY" for short, was proposed to handle ordinary arbitrary-deadline sporadic task systems (without suspensions). (Note that GY is the only prior work that considers multiprocessor RTA techniques for arbitrarydeadline task systems.) In GY, the methodology used for the constrained-deadline case is extended for dealing with the arbitrary-deadline case by recursively solving an RTA equation. This recursive process could iterate many times depending on task parameters, and may not terminate in some rare cases. On the other hand, due to the fact that our analysis used a more suitable interval analysis framework for arbitrary-deadline task systems ${ }^{5}$ (which applies to constrained-deadline task systems as well), for any task in an ordinary sporadic task systems (without suspensions), its response-time bound can be found by solving the RTA equation (14) only once and our RTA process always

\footnotetext{
${ }^{5}$ Specifically, we analyzed the eligible time $t_{e}$ of our job of interest $\tau_{l, j}$, instead of its release time $r_{l, j}$ as done in [8]. In this way, when computing workload and interference, we already considered the case where job $\tau_{l, k}$ where $k<j$ might complete beyond $r_{l, j}$ if $d_{l}>p_{l}$ holds. On the contrary, in GY, the analyzed interval is extended to include all prior jobs that may complete beyond $r_{l, j}$ and an RTA equation is recursively solved to find the response-time bound.
}

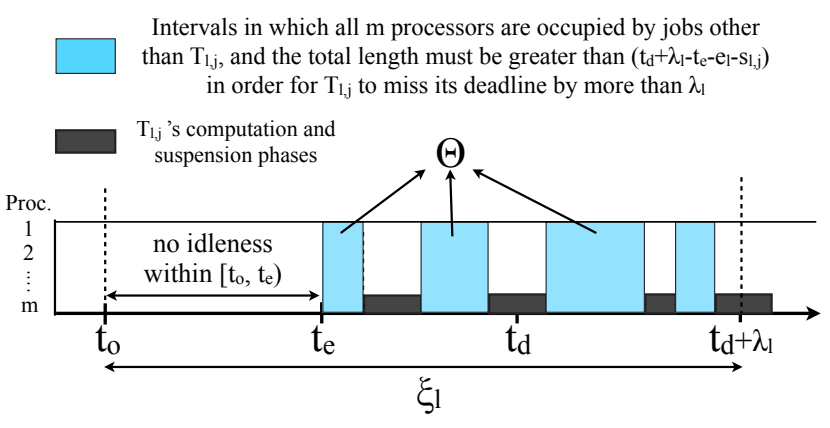

Fig. 4: A job $\tau_{l, j}$ of task $\tau_{l}$ becomes eligible at $t_{e}$ and misses its deadline at $t_{d}$ by more than $\lambda_{l}$. $t_{o}$ is the earliest time instant at or before $t_{e}$ such that there is no idleness in $\left[t_{o}, t_{e}\right)$.

terminates. As shown by experiments presented in Sec. V, our analysis has better runtime performance than GY.

\section{GEDF Schedulability Test}

In this section, we present a GEDF schedulability test for SSS task systems. Our goal is to identify sufficient conditions for ensuring that each task $\tau_{i}$ cannot miss any deadlines by more than its predefined tardiness threshold, $\lambda_{i}$. These conditions must be checked for each of the $n$ tasks in $\tau$.

Let $S$ be a GEDF schedule of $\tau$ such that a job $\tau_{l, j}$ of task $\tau_{l}$ is the first job in $S$ to miss its deadline at $t_{d}=d_{l, j}$ by more than its predefined tardiness threshold $\lambda_{l}$, as shown in Fig. 4. Under GEDF, jobs with lower priorities than $\tau_{l, j}$ do not affect the scheduling of $\tau_{l, j}$ and jobs with higher priorities than $\tau_{l, j}$, so we will henceforth discard from $S$ all jobs with priorities lower than $\tau_{l, j}$.

Similar to Sec. III, we extend the analyzed interval from $\tau_{l, j}$ 's eligible time $t_{e}$ (see Def. 3) to an earlier time instant $t_{o}$ as defined below.

Definition 7. $t_{o}$ denotes the earliest time instant at or before $t_{e}$ such that there is no idleness in $\left[t_{o}, t_{e}\right)$.

Our goal now is to identify conditions necessary for $\tau_{l, j}$ to miss its deadline by more than $\lambda_{l}$; i.e., for $\tau_{l, j}$ to execute its computation and suspension phases for strictly fewer than $e_{l}+s_{l, j}$ time units over $\left[t_{e}, t_{d}+\lambda_{l}\right)$. This can happen only if all $m$ processors execute jobs other than $\tau_{l, j}$ for strictly more than $\left(t_{d}+\lambda_{l}-t_{e}\right)-\left(e_{l}+s_{l, j}\right)$ time units (i.e., at least $t_{d}+\lambda_{l}-t_{e}-e_{l}-s_{l, j}+1$ time units) over $\left[t_{e}, t_{d}+\lambda_{l}\right.$ ) (for otherwise, $\tau_{l, j}$ would complete by $\left.t_{d}+\lambda_{l}\right)$, as illustrated in Fig. 4. For conciseness, let

$$
\xi_{l}=t_{d}+\lambda_{l}-t_{o} .
$$

Definition 8. Let $\Theta$ denote a subset of the set of intervals within $\left[t_{e}, t_{d}+\lambda_{l}\right)$, where $\tau_{l, j}$ does not execute or suspend, 
such that the cumulative length of $\Theta$ is exactly $t_{d}+\lambda_{l}-$ $t_{e}-e_{l}-s_{l, j}+1$ over $\left[t_{e}, t_{d}+\lambda_{l}\right)$. As seen in Fig. $4, \Theta$ may not be contiguous.

By Def. 8, the length of the intervals in $\left[t_{o}, t_{e}\right) \cup \Theta$ is given by $t_{e}-t_{o}+t_{d}+\lambda_{l}-t_{e}-e_{l}-s_{l, j}+1=t_{d}+\lambda_{l}-$

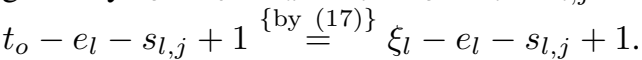

For each task $\tau_{i}$, let $W\left(\tau_{i}\right)$ denote the contribution of $\tau_{i}$ to the work done in $S$ during $\left[t_{o}, t_{e}\right) \cup \Theta$. In order for $\tau_{l, j}$ to miss its deadline, it is necessary that the total amount of work that executes over $\left[t_{o}, t_{e}\right) \cup \Theta$ satisfies

$$
\sum_{\tau_{i} \in \tau} W\left(\tau_{i}\right)>m \cdot\left(\xi_{l}-e_{l}-s_{l, j}\right) .
$$

This follows from the observation that all $m$ processors are, by Defs. 7 and 8 , completely busy executing work over the $\xi_{l}-e_{l}-s_{l, j}+1$ time units in the interval $\left[t_{o}, t_{e}\right) \cup \Theta$.

Condition (18) is a necessary condition for $\tau_{l, j}$ to miss its deadline by more than $\lambda_{l}$. Thus, in order to show that $\tau$ is GEDF-schedulable, it suffices to demonstrate that Condition (18) cannot be satisfied for any task $\tau_{l}$ for any possible values of $\xi_{l}$ and $s_{l, j}$.

We now construct a schedulability test using Condition (18) as follows. In Sec. IV-A, we first derive an upper bound for the term $\sum_{\tau_{i} \in \tau} W\left(\tau_{i}\right)$ in the LHS of Condition (18). Then, in Sec. IV-B, we compute possible values of the term $m \cdot\left(\xi_{l}-e_{l}-s_{l, j}\right)$ in the RHS of Condition (18). Later, in Sec. IV-C, a schedulability test is derived based on these results.

\section{A. Upper-Bounding $\sum_{\tau_{i} \in \tau} W\left(\tau_{i}\right)$}

In this section, we derive an upper bound on $\sum_{\tau_{i} \in \tau} W\left(\tau_{i}\right)$, by first upper-bounding $W\left(\tau_{i}\right)$ for each task $\tau_{i}$ and then summing these per-task upper bounds.

In the following, we compute upper bounds on $W\left(\tau_{i}\right)$. If $\tau_{i}$ has no carry-in job (defined in Def. 5), then let $W_{n c}\left(\tau_{i}\right)$ denote this upper bound; otherwise, let $W_{c}\left(\tau_{i}\right)$ denote the upper bound. Since $\tau_{l, j}$ is the first job that misses its deadline at $t_{d}$ by more than its corresponding tardiness threshold, we have

$$
f_{l, j-1} \leq d_{l, j-1}+\lambda_{l} \leq t_{d}-p_{l}+\lambda_{l} .
$$

The following lemma bounds the length of time interval $\left[t_{o}, t_{e}\right)$.

Lemma 5. $t_{e}-t_{o} \leq \max \left(\xi_{l}-\lambda_{l}-d_{l}, \xi_{l}-p_{l}\right)$.

$$
\begin{aligned}
& \text { Proof: } t_{e}-t_{o} \quad \text { by } \stackrel{\text { Def. } 3\}}{=} \max \left(r_{l, j}, f_{l, j-1}\right)- \\
& t_{o} \stackrel{\{\text { by }}{\leq} \max \left(t_{d}-d_{l}, t_{d}-p_{l}+\lambda_{l}\right)-t_{o}=\max \left(t_{d}-\right. \\
& \left.d_{l}-t_{o}, t_{d}-p_{l}+\lambda_{l}-t_{o}\right) \stackrel{\{\text { by }}{=}{ }^{(17)\}} \max \left(\xi_{l}-\lambda_{l}-d_{l}, \xi_{l}-p_{l}\right) \text {. }
\end{aligned}
$$

If a task $\tau_{i}$ has no carry-in job, then the total amount of work that must execute over $\left[t_{o}, t_{e}\right) \cup \Theta$ is generated by jobs

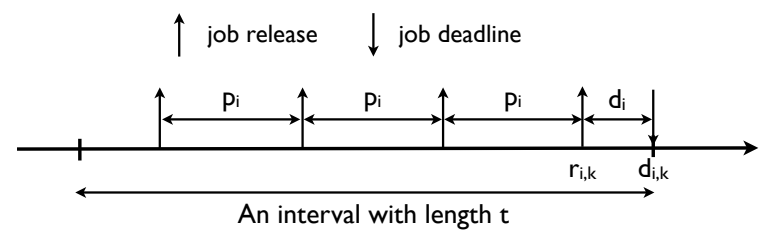

Fig. 5: DBF for self-suspending tasks.

of $\tau_{i}$ arriving in, and having deadlines within, the interval $\left[t_{o}, t_{d}\right]$. The following lemma, which was originally proved for ordinary sporadic task systems [4], applies to SSS task systems as well.

Lemma 6. The maximum cumulative execution requirement by jobs of an SSS task $\tau_{i}$ that both arrive in, and have deadlines within, any interval of length $t$ is given by demand bound function

$$
D B F\left(\tau_{i}, t\right)=\max \left(0,\left(\left\lfloor\frac{t-d_{i}}{p_{i}}\right\rfloor+1\right) \cdot e_{i}\right) .
$$

Proof: Because we restrict attention to jobs of $\tau_{i}$ that have releases and deadlines within the considered interval of length $t$, and suspensions do not occupy any processor, the required total work of $\tau_{i}$ can be bounded by considering the scenario in which some job $\tau_{i, k}$ of $\tau_{i}$ has a deadline at the end of the interval and jobs are released periodically. This scenario is illustrated in Fig. 5. There are at most $\left\lfloor\frac{t-d_{i}}{p_{i}}\right\rfloor$ jobs that are released and have deadlines within the interval other than $\tau_{i, k}$. Thus, the maximum cumulative execution requirement by jobs of $\tau_{i}$ is given by $D B F\left(\tau_{i}, t\right)=\max \left(0,\left(\left\lfloor\frac{t-d_{i}}{p_{i}}\right\rfloor+1\right) \cdot e_{i}\right)$, which is the same as the DBF for ordinary sporadic tasks (i.e., without suspensions). This is due to the fact that suspensions do not contribute to the execution requirement.

The lemma below computes $W_{n c}\left(\tau_{i}\right)$ using DBF.

\section{Lemma 7.}

$W_{n c}\left(\tau_{i}\right)= \begin{cases}\min \left(D B F\left(\tau_{i}, \xi_{l}-\lambda_{l}\right),\right. & \\ \left.\xi_{l}-e_{l}-s_{l, j}+1\right) & \text { if } i \neq l \\ \min \left(D B F\left(\tau_{l}, \xi_{l}-\lambda_{l}\right)-e_{l},\right. & \\ \left.\max \left(\xi_{l}-\lambda_{l}-d_{l}, \xi_{l}-p_{l}\right)\right) & \text { if } i=l\end{cases}$

Proof: Depending on the relationship between $i$ and $l$, there are two cases to consider.

Case $i \neq l$. The total amount of work contributed by $\tau_{i}$ that must execute over $\left[t_{o}, t_{e}\right) \cup \Theta$ cannot exceed the total length of the intervals in $\left[t_{o}, t_{e}\right) \cup \Theta$, which is $\xi_{l}-$ $e_{l}-s_{l, j}+1$. Furthermore, the total work that needs to be bounded must have releases and deadlines within the interval $\left[t_{o}, t_{d}\right]$, which by (17) is of length $\xi_{l}-\lambda_{l}$. By Lemma 6, this total work is at most $\operatorname{DBF}\left(\tau_{i}, \xi_{l}-\lambda_{l}\right)$.

Case $i=l$. As in the previous case, the total work is at most $D B F\left(\tau_{l}, \xi_{l}-\lambda_{l}\right)$. However, in this case, since $\tau_{l, j}$ does not execute within $\left[t_{o}, t_{e}\right) \cup \Theta$, we can subtract its execution requirement, which is $e_{l}$, from $\operatorname{DBF}\left(\tau_{l}, \xi_{l}-\right.$ 
$\left.\lambda_{l}\right)$. Also, this contribution cannot exceed the length of the interval $\left[t_{o}, t_{e}\right)$, which by Lemma 5 is at $\operatorname{most} \max \left(\xi_{l}-\right.$ $\left.\lambda_{l}-d_{l}, \xi_{l}-p_{l}\right)$.

We now consider the case where $\tau_{i}$ has a carry-in job. The following lemma, which computes $W_{c}\left(\tau_{i}\right)$, is proved similarly to Lemma 7.

\section{Lemma 8.}

$$
W_{c}\left(\tau_{i}\right)= \begin{cases}\min \left(\Delta\left(\tau_{i}, \xi_{l}-\lambda_{l}+\lambda_{i}\right),\right. & \\ \left.\xi_{l}-e_{l}-s_{l, j}+1\right) & \text { if } i \neq l \\ \min \left(\Delta\left(\tau_{l}, \xi_{l}\right)-e_{l},\right. & \\ \max \left(\xi_{l}-\lambda_{l}-d_{l}, \xi_{l}-p_{l}\right) . & \text { if } i=l\end{cases}
$$

Proof: The total work of $\tau_{i}$ in this case can be upperbounded by considering the scenario in which some job of $\tau_{i}$ has a deadline at $t_{d}$ and jobs of $\tau_{i}$ are released periodically, as illustrated in Fig. 6. Depending on the relationship between $i$ and $l$, we have two cases to consider.

Case $i \neq l$. Let $\tau_{i, k}$ be the first job such that $r_{i, k}<t_{o}$ and

$$
d_{i, k}+\lambda_{i}>t_{o},
$$

i.e., $\tau_{i, k}$ is the first job of $\tau_{i}$ (potentially tardy) that may execute during $\left[t_{o}, t_{d}\right)$ and is released before $t_{o}$ (note that if $\tau_{i, k}$ does not exist then $\tau_{i}$ would have no carry-in job). Since jobs are released periodically,

$$
t_{d}-d_{i, k}=x \cdot p_{i}
$$

holds for some integer $x$.

The demand for jobs of $\tau_{i}$ in this case is thus bounded by the demand due to $x$ jobs that have deadlines at or before $t_{d}$ and are released at or after $r_{i, k}+p_{i}$, plus the demand imposed by the job $\tau_{i, k}$, which cannot exceed the smaller of $e_{i}$ and the length of the interval $\left[t_{o}, d_{i, k}+\lambda_{i}\right)$, which by (21) is $t_{d}-x \cdot p_{i}+\lambda_{i}-t_{o} \stackrel{\text { by }}{=}{ }^{(17)\}} \xi_{l}-\lambda_{l}+\lambda_{i}-x \cdot p_{i}$. Thus, we have

$$
W_{c}\left(\tau_{i}\right)=x \cdot e_{i}+\min \left(e_{i}, \xi_{l}-\lambda_{l}+\lambda_{i}-x \cdot p_{i}\right) .
$$

To find $x$, by (21), we have $\left.x=\frac{t_{d}-d_{i, k}}{p_{i}} \stackrel{\text { by }}{<}(20)\right\}$ $\frac{t_{d}-t_{o}+\lambda_{i}}{p_{i}}\left\{\right.$ by $\stackrel{(17)\}}{\underline{\xi_{l}-\lambda_{l}+\lambda_{i}}}$. For conciseness, let $\pi=$ $\frac{p_{i}}{\xi_{l}-\lambda_{l}+\lambda_{i}}$. Thus, $x<\frac{\pi_{p}}{p_{i}}$ holds. If $\pi \bmod p_{i}=0$, then $x \leq$ $\frac{\pi}{p_{i}}-1=\left\lceil\frac{\pi}{p_{i}}\right\rceil-1$, otherwise, $x \leq\left\lfloor\frac{\pi}{p_{i}}\right\rfloor=\left\lceil\frac{\pi}{p_{i}}\right\rceil-1$. Thus, a general expression for $x$ can be given by $x \leq\left\lceil\frac{\pi}{p_{i}}\right\rceil-1$.

By (22), the maximum value for $W_{c}\left(\tau_{i}\right)$ can be obtained when $x=\left\lceil\frac{\pi}{p_{i}}\right\rceil-1$. Setting this expression for $x$ into (22), we have $W_{c}\left(\tau_{i}\right)=\left(\left\lceil\frac{\pi}{p_{i}}\right\rceil-1\right) \cdot e_{i}+\min \left(e_{i}, \pi-\left\lceil\frac{\pi}{p_{i}}\right\rceil \cdot p_{i}+\right.$

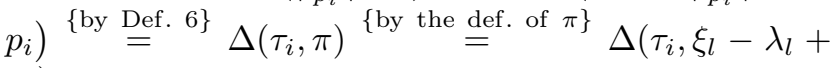
$\left.\lambda_{i}\right)$.

Moreover, this total demand cannot exceed the total length of the intervals in $\left[t_{o}, t_{e}\right) \cup \Theta$, which is $\xi_{l}-e_{l}-$ $s_{l, j}+1$.

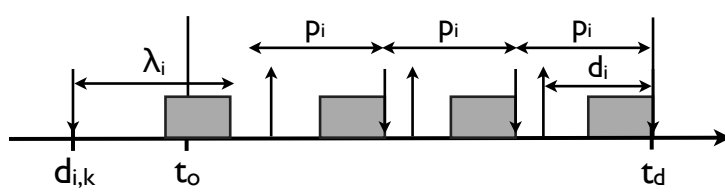

Fig. 6: Computing $W_{c}\left(\tau_{i}\right)$.

Case $i=l$. Repeating the reasoning from the previous case, we find that the total demand of jobs of $\tau_{l}$ with deadlines at most $t_{d}$ is at most $\Delta\left(i, \xi_{l}-\lambda_{l}+\lambda_{i}\right)=\Delta\left(\tau_{i}, \xi_{l}\right)$. Since $\tau_{l, j}$ does not execute within $\left[t_{o}, t_{e}\right) \cup \Theta$, we subtract its execution requirement, which is $e_{l}$, from $\Delta\left(\tau_{i}, \xi_{l}\right)$. Also, this contribution cannot exceed the length of the interval $\left[t_{o}, t_{e}\right)$, which by Lemma 5 is $\max \left(\xi_{l}-\lambda_{l}-d_{l}, \xi_{l}-p_{l}\right)$.

Upper-bounding $\sum_{\tau_{i} \in \tau} W\left(\tau_{i}\right)$. Similar to the discussion in Sec. III, by Def. 7, either $t_{o}=0$, in which case no task has a carry-in job, or some processor is idle in $\left[t_{o}-1, t_{o}\right)$, in which at most $m-1$ computational tasks are active at $t_{o}-1$. Thus, at most $\min \left(m-1, n_{e}\right)$ computational tasks can have a carry-in job. However, since suspensions do not occupy any processor, each self-suspending task may be active at $t_{o}-1$ and have a job that is suspended at $t_{o}$. Thus, in the worst case, all $n_{s}$ self-suspending tasks can have carry-in jobs. Consequently, there are at most $n_{s}$ self-suspending tasks and $\min \left(m-1, n_{e}\right)$ computational tasks that contribute $W_{c}\left(\tau_{i}\right)$ work, and the remaining $\max \left(0, n_{e}-m+1\right)$ computational tasks must contribute to $W_{n c}\left(\tau_{i}\right)$. Thus, self-suspending tasks can contribute at most $\sum_{\tau_{i} \in \tau^{s}} \max \left(W_{n c}\left(\tau_{i}\right), W_{c}\left(\tau_{i}\right)\right)$ work to $\sum_{\tau_{i} \in \tau} W\left(\tau_{i}\right)$. Let $\delta_{\tau_{i} \in \tau^{e}}^{\min \left(m-1, n_{e}\right)}$ denote the $\min \left(m-1, n_{e}\right)$ greatest values of $\max \left(0, W_{c}\left(\tau_{i}\right)-W_{n c}\left(\tau_{i}\right)\right)$ for any computational task $\tau_{i}$. Then computational tasks can contribute at most $\sum_{\tau_{j} \in \tau^{e}} W_{n c}\left(\tau_{j}\right)+\delta_{\tau_{k} \in \tau^{e}}^{\min \left(m-1, n_{e}\right)}$ work to $\sum_{\tau_{i} \in \tau} W\left(\tau_{i}\right)$. Therefore, by summing up the work contributed by both self-suspending tasks and computational tasks, we can bound $\sum_{\tau_{i} \in \tau} W\left(\tau_{i}\right)$ by $\sum_{\tau_{i} \in \tau^{s}} \max \left(W_{n c}\left(\tau_{i}\right)\right.$, $\left.W_{c}\left(\tau_{i}\right)\right)+\sum_{\tau_{j} \in \tau^{e}} W_{n c}\left(\tau_{j}\right)+\delta_{\tau_{k} \in \tau^{e}}^{\min \left(m-1, n_{e}\right)}$.

Similar to the discussion in Sec. III, the time complexity for computing $W_{c}\left(\tau_{i}\right), W_{n c}\left(\tau_{i}\right)$, and $W_{c}\left(\tau_{i}\right)-$ $W_{n c}\left(\tau_{i}\right)$ is $O(n)$. Also, by using a linear-time selection technique from [6], the time complexity for computing $\delta_{\tau_{k} \in \tau^{e}}^{\min \left(m-1, n_{e}\right)}$ is $O(n)$. Thus, the time complexity to upperbound $\sum_{\tau_{i} \in \tau} W\left(\tau_{i}\right)$ as above is $O(n)$.

\section{B. Finding Values of $\xi_{l}$ and $s_{l, j}$}

So far we have upper-bounded the LHS of Condition (18). Recall that our goal is to test Condition (18) for a violation for all possible values of $\xi_{l}$ and $s_{l, j}$. The following theorem shows that the range of possible values of $\xi_{l}$ that need to be tested can be limited. Let $e_{\text {sum }}$ be the sum of the execution costs for all tasks in $\tau$. For conciseness, let $\phi=$ 
$m \cdot\left(e_{l}+s_{l, j}\right)-\lambda_{l} \cdot u_{\text {sum }}+\sum_{\tau_{i} \in \tau} \lambda_{i} \cdot u_{i}+e_{\text {sum }}$.

Theorem 2. If Condition (18) is satisfied for $\tau_{l}$, then it is satisfied for some $\xi_{l}$ satisfying

$$
\min \left(d_{l}+\lambda_{l}, p_{l}\right) \leq \xi_{l}<\frac{\phi}{m-u_{\text {sum }}},
$$

provided $u_{\text {sum }}<m$

Proof: By Lemmas 6 and 7, $W_{n c}\left(\tau_{i}\right) \leq\left\lfloor\frac{\xi_{l}-\lambda_{l}}{p_{i}}\right\rfloor \cdot e_{i}+e_{i}$ holds. By Lemma 8 and Def. 6, $W_{c}\left(\tau_{i}\right) \leq\left\lfloor\frac{\xi_{l}-\lambda_{l}+\lambda_{i}}{p_{i}}\right\rfloor \cdot e_{i}+$ $e_{i}$ holds. Thus, the LHS of Condition (18) is no greater than $\sum_{\tau_{i} \in \tau}\left(\left\lfloor\frac{\xi_{l}-\lambda_{l}+\lambda_{i}}{p_{i}}\right\rfloor \cdot e_{i}+e_{i}\right)$. Assuming Condition (18) is satisfied, we have

$$
\begin{array}{ll} 
& \sum_{\tau_{i} \in \tau} W\left(\tau_{i}\right)>m \cdot\left(\xi_{l}-e_{l}-s_{l, j}\right) \\
\Rightarrow \quad & \text { upper-bounding } \left.\sum_{\tau_{i} \in \tau} W\left(\tau_{i}\right) \text { as above }\right\} \\
& \sum_{\tau_{i} \in \tau}\left(\left\lfloor\frac{\xi_{l}-\lambda_{l}+\lambda_{i}}{p_{i}}\right\rfloor \cdot e_{i}+e_{i}\right)>m \cdot\left(\xi_{l}-e_{l}-s_{l, j}\right) \\
\Rightarrow \quad & \{\text { removing the floor }\} \\
& \sum_{\tau_{i} \in \tau}\left(\left(\xi_{l}-\lambda_{l}+\lambda_{i}\right) \cdot u_{i}+e_{i}\right)>m \cdot\left(\xi_{l}-e_{l}-s_{l, j}\right) \\
\Rightarrow \quad & \{\text { rearranging }\} \\
& \xi_{l} \cdot u_{\text {sum }}-\lambda_{l} \cdot u_{\text {sum }}+\sum_{\tau_{i} \in \tau} \lambda_{i} \cdot u_{i}+e_{\text {sum }} \\
& >m \cdot \xi_{l}-m \cdot e_{l}-m \cdot s_{l, j} \\
\Rightarrow \quad & \xi_{l}<\frac{\phi}{m-u_{\text {sum }}},
\end{array}
$$

provided $u_{\text {sum }}<m$.

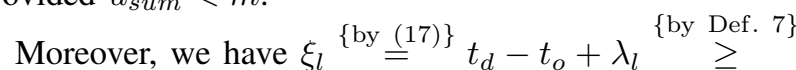
$t_{d}-t_{e}+\lambda_{l} \stackrel{\left\{\text { by } \stackrel{\text { Def. } 3\}}{=} t_{d}-\max \left(r_{l, j}, f_{l, j-1}\right)+\lambda_{l} \stackrel{\{\text { by }}{\geq} \geq\right.}{\geq}$ $t_{d}-\max \left(t_{d}-d_{l}, t_{d}-p_{l}+\lambda_{l}\right)+\lambda_{l}=\min \left(d_{l}, p_{l}-\lambda_{l}\right)+\lambda_{l}=$ $\min \left(d_{l}+\lambda_{l}, p_{l}\right)$.

Possible values for $s_{l, j}$. By Lemmas 7 and 8, $\sum_{\tau_{i} \in \tau} W\left(\tau_{i}\right)$, which is the LHS of Condition (18), depends on the value of $s_{l, j}$ non-monotonically. Moreover, by Theorem $2, \xi_{l}$ also depends on the value of $s_{l, j}$ (recall $\phi)$. Thus, it is necessary to test all possible values of $s_{l, j}$, which are $\left\{0,1,2, \ldots, s_{l}\right\}$.

\section{Schedulability Test}

Theorem 3. Task system $\tau$ is GEDF-schedulable on $m$ processors if for all tasks $\tau_{l}$ and all values of $\xi_{l}$ satisfying (23),

$$
\begin{gathered}
\sum_{\tau_{i} \in \tau} \max \left(W_{n c}\left(\left(\tau_{i}\right), W_{c}\left(\tau_{i}\right)\right)+\sum_{\tau_{j} \in \tau^{e}} W_{n c}\left(\tau_{j}\right)\right. \\
\left.+\delta_{\tau_{k} \in \tau^{e}}^{\min \left(m-1, n_{e}\right)}\right) \leq m \cdot\left(\xi_{l}-e_{l}-s_{l, j}\right)
\end{gathered}
$$

holds for every value of $s_{l, j} \in\left\{0,1,2, \ldots, s_{l}\right\}$.

By Theorem 2, we can test Condition (24) in time pseudo-polynomial in the task parameters.

\section{Experiments}

In this section, we describe experiments conducted using randomly-generated task sets to evaluate the performance of the proposed schedulability tests. In these experiments, several aspects of our analysis were investigated. In the following, we denote our GEDF and GFP schedulability tests as "Our-EDF" and "Our-FP," respectively.

HRT effectiveness. We evaluated the effectiveness of the proposed techniques for HRT SSS task systems by comparing Our-EDF and Our-FP to the suspension-oblivious approach denoted "SC" combined with the tests in [2] and [8], which we denote "Bar" and (as noted earlier) "GY," respectively. That is, after transforming all SSS tasks into ordinary sporadic tasks (no suspensions) using SC, we applied Bar and GY, which are the best known schedulability tests for GEDF and GFP, respectively. In [2], Bar was shown to overcome a major deficiency (i.e., the $O(n)$ carry-in work) of prior GEDF analysis. In [8], GY was shown to be superior to all prior analysis for ordinary task systems available at that time. Moreover, since partitioning approaches have been shown to be generally superior to global approaches on multiprocessors [1], we compared our test to SC combined with the partitioning approach proposed in [3], which we denoted "FB-Par". FBPar is considered to be the best partitioning approach for constrained-deadline sporadic task systems.

SRT effectiveness. We evaluated the effectiveness of the proposed techniques for SRT SSS task systems with predefined tardiness threshold by comparing them to SC combined with the test proposed in [10], which we denote "LA." LA is the only prior schedulability test for SRT ordinary task systems with predefined tardiness thresholds.

Impact of carry-in work. To evaluate the impact brought by $O(n)$ carry-in work on our analysis, we compared the HRT schedulability for SSS task systems using our analysis to that obtained by applying the analysis proposed in [5], which we denoted "BC," to an otherwise equivalent task system with no suspensions. In [5], BC was shown to be superior to all prior analysis assuming $O(n)$ carry-in work available at that time.

Runtime performance. Finally, we evaluated the effectiveness and the runtime performance of Our-FP for ordinary arbitrary-deadline sporadic task systems (with no suspensions) by comparing it to GY.

In our experiments, SSS task sets were generated based upon the methodology proposed by Baker in [1]. Integral task periods were distributed uniformly over [10ms,100ms]. Per-task utilizations were uniformly distributed in [0.01, 0.3]. Task execution costs were calculated from periods and utilizations. For any task $\tau_{i}$ in any generated task set, $d_{i} / p_{i}$ was varied within $[1,2]$ for the arbitrary-deadline case and within $\left[\max \left(0.7, \frac{e_{i}+s_{i}}{p_{i}}\right), 1\right]$ for the constraineddeadline case, and the tardiness threshold $\lambda_{i}$ was varied uniformly within $\left[0,2 \cdot p_{i}\right]$ for SRT tasks. The suspension 


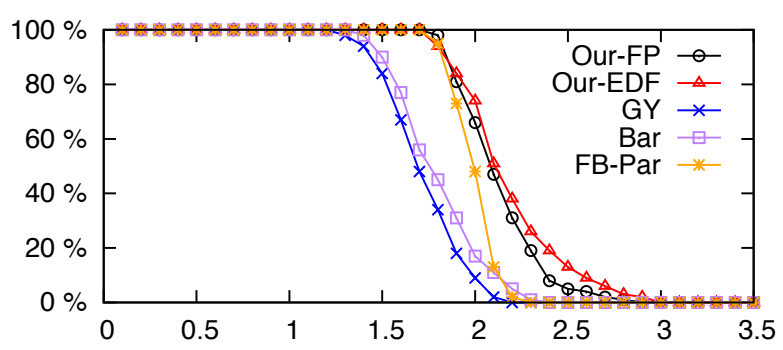

(a) HRT: short suspensions

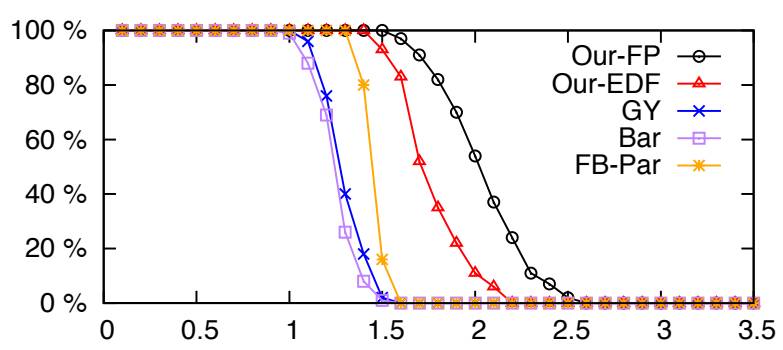

(c) HRT: moderate suspensions

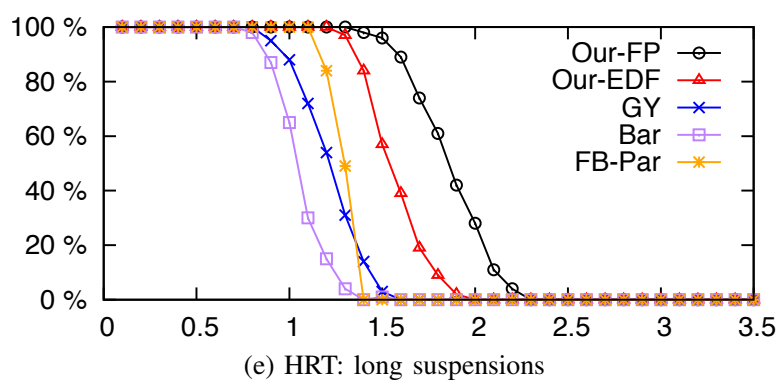

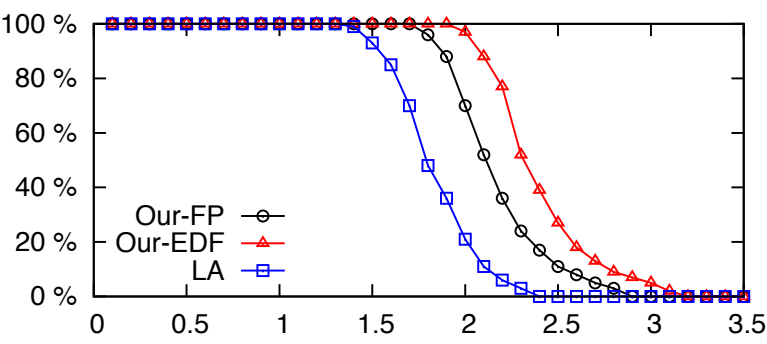

(b) SRT: short suspensions

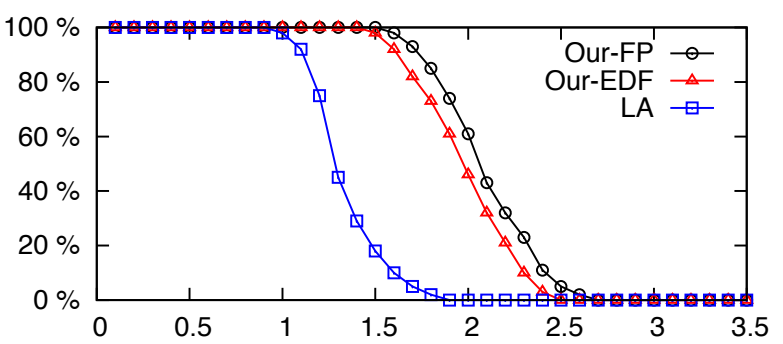

(d) SRT: moderate suspensions

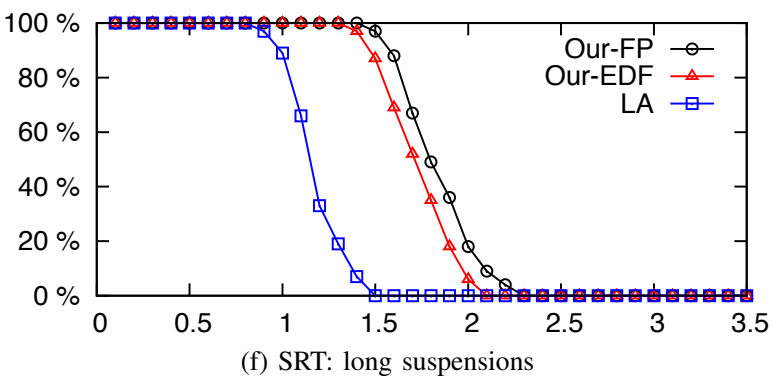

Fig. 7: HRT and SRT results. $u_{i} \in[0.01,0.3], d_{i} \in\left[\max \left(0.7 \cdot p_{i}, e_{i}+s_{i}\right), p_{i}\right]$.

length for any task $\tau_{i}$ was generated by varying $s_{i} / e_{i}$ as follows: 0.5 (short suspension length), 1 (moderate suspension length), and 1.5 (long suspension length). Task sets were generated for $m=4$ processors, as follows. A cap on overall utilization was systematically varied within $[1,1.1,1.2, \ldots, 3.9,4]$. For each combination of utilization cap and suspension length, we generated 1,000 SSS task sets. Each such SSS task set was generated by creating SSS tasks until total utilization exceeded the corresponding utilization cap, and by then reducing the last task's utilization so that the total utilization equalled the utilization cap. For GFP scheduling, priorities were assigned on a global deadline-monotonic basis. In all figures presented in this section, the $x$-axis denotes the utilization cap and the $y$ axis denotes the fraction of generated task sets that were schedulable.

Fig. 7 shows HRT and SRT schedulability results for constrained-deadline SSS task sets achieved by using OurEDF, Our-FP, Bar, GY, and FB-Par. As seen, for both the
HRT and the SRT cases, Our-EDF and Our-FP improve upon the other tested alternatives. Notably, Our-EDF and Our-FP consistently yield better schedulability results than the partitioning approach FB-Par. This is due to the fact that, after treating suspensions as computation, FB-Par suffers from bin-packing-related utilization loss. Moreover, as the suspension length increases, such performance improvement also increases. This is because treating suspension as computation becomes more pessimistic as the suspension length increases. This result suggests that a task's suspensions do not negatively impact the schedulability of other tasks as much as computation does.

Fig. 8 shows HRT schedulability results for constraineddeadline SSS task sets achieved by using Our-FP and by applying BC to otherwise equivalent task sets with no suspensions. In Fig. 8, "Our-FP-S” (respectively, "Our-FPM") represents schedulability results achieved by Our-FP for the task sets (which are originally generated for BC with no suspensions) after adding suspensions by setting 


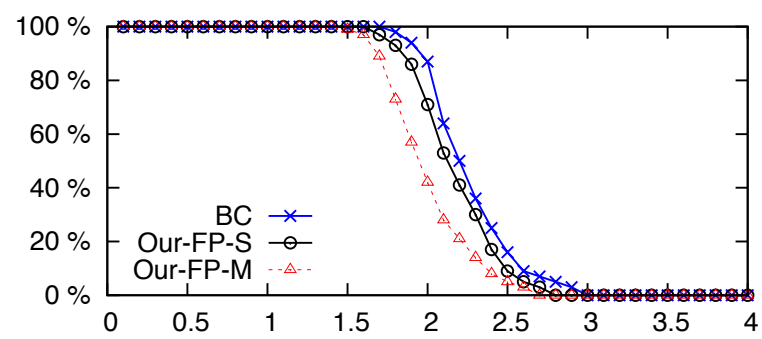

Fig. 8: HRT results compared with BC.

$\frac{s_{i}}{e_{i}}=0.2$ (respectively, $\frac{s_{i}}{e_{i}}=0.5$ ). It can be seen that OurFP yields schedulability results that are very close to that achieved by BC. For task sets with $\frac{s_{i}}{e_{i}}=0.2$, Our-FP and $\mathrm{BC}$ achieved almost identical schedulability results. This shows that the negative impact brought by suspensions is mainly caused by forcing $O(n)$ carry-in work.

Fig. 9 shows HRT schedulability results for arbitrarydeadline ordinary task systems (with no suspensions) achieved by using Our-FP and GY. In this experiment, for each choice of the utilization cap, 10,000 task sets are generated. As seen, Our-FP slightly improves upon GY. Moreover, Fig. 9 also shows the total time for running this entire experiment for Our-FP and GY. As seen, Our-FP runs much faster $(>10 \times)$ than GY, due to the fact that Our-FP can find any task's response time by solving the RTA equation only once.

\section{Conclusion}

We have presented HRT and SRT (with predefined tardiness thresholds) multiprocessor schedulability tests for arbitrary-deadline SSS task systems under both GFP and GEDF scheduling. In experiments presented herein, our suspension-aware analysis results significantly improve upon the suspension-oblivious approach SC. Moreover, our analysis shows that by applying an interval analysis framework tailored to the arbitrary-deadline case, arbitrarydeadline task systems (both ordinary and self-suspending ones) can be analyzed much more efficiently. Experiments indicate that our analysis has much better runtime performance than the prior approach proposed in [8].

In future work, it would be interesting to investigate more precise and practical suspension patterns. That is, instead of assuming that each task's suspensions are simply upper-bounded and will not be interfered with by other tasks' suspensions, it would be interesting to allow a task's suspension lengths to be affected by other tasks' suspensions (e.g., due to contention when multiple tasks simultaneously access the same shared resource).

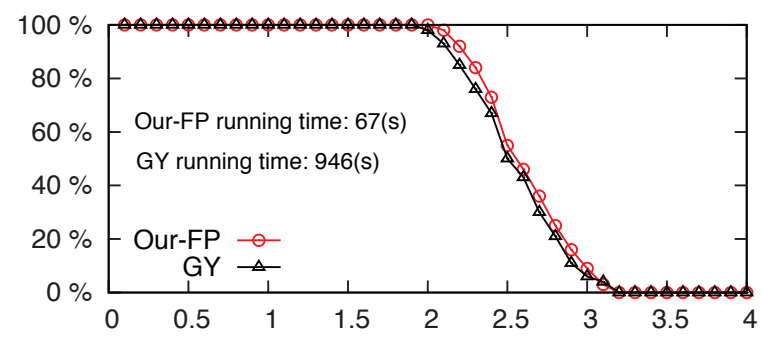

Fig. 9: HRT results compared with GY.

\section{References}

[1] T. Baker. A comparison of global and partitioned EDF schedulability tests for multiprocessors. In Technical Report TR-051101, Florida State University, 2005.

[2] S. Baruah. Techniques for multiprocessor global schedulability analysis. In Proc. of the 28th RTSS, pp. 119-128, 2007.

[3] S. Baruah and N. Fisher. The partitioned multiprocessor scheduling of sporadic task systems. In Proc. of the 26th RTSS, pp. 321-329, 2005.

[4] S. Baruah, A. Mok, and L. Rosier. Preemptively scheduling hardreal-time sporadic tasks on one processor. In Proc. of the 11th RTSS, pp. 182-190, 1990.

[5] M. Bertogna and M. Cirinei. Response-time analysis for globally scheduled symmetric multiprocessor platforms. In Proc. of the 28th RTSS, pp. 149-160, 2007.

[6] M. Blum, R. W. Floyd, V. Pratt, R. L. Rivest, and R. E. Trarjan. Time bounds for selection. JCSS, (4): 448-461, 1973.

[7] G. Elliott and J. Anderson. Real-World Constraints of GPUs in RealTime Systems. In Proceedings of the First International Workshop on Cyber-Physical Systems, Networks, and Applications, pp. 48-54, 2011.

[8] N. Guan, M. Stigge, W. Yi, and G. Yu. New response time bounds for fixed priority multiprocessor scheduling. In Proc. of the 30th RTSS, pp. 387-397, 2009.

[9] S. Lee and B. Moon. Design of flash-based DBMS: an in-page logging approach. In Proc. of the 2007 ACM SIGMOD Conf. on Management of Data, pp. 55-66, 2007.

[10] H. Leontyev and J. Anderson. A unified hard/soft real-time schedulability test for global EDF multiprocessor scheduling. In Proc. of the 29th RTSS, pp. 375-384, 2008.

[11] C. Liu and J. Anderson. Improving the schedulability of sporadic self-suspending soft real-time multiprocessor task systems. In Proc. of the 16th IEEE Int'l Conf. on Embedded and Real-Time Computing Sys. and Apps., pp. 14-23, 2010.

[12] C. Liu and J. Anderson. Scheduling suspendable, pipelined tasks with non-preemptive sections in soft real-time multiprocessor systems. In Proc. of the 16th IEEE Real-Time and Embedded Tech. and Apps. Symp., pp. 23-32, 2010.

[13] C. Liu and J. Anderson. An O(m) analysis technique for supporting real-time self-suspending task systems. In Proc. of the 33rd RTSS, pp. 373-382, 2012.

[14] C. Liu and J. Anderson. Task scheduling with self-suspensions in soft real-time multiprocessor systems. In Proc. of the 30th RTSS, pp. 425-436, 2009.

[15] C. Liu, J. Li, W. Huang, J. Rubio, E. Speight, and X. Lin. Power-efficient time-sensitive mapping in cpu/gpu heterogeneous systems. In Proc. of the 21st International Conference on Parallel Architectures and Compilation Techniques, pp. 23-32, 2012.

[16] J. Liu. Real-time systems. Prentice Hall, 2000.

[17] F. Ridouard, P. Richard, and F. Cottet. Negative results for scheduling independent hard real-time tasks with self-suspensions. In Proc. of the 25th RTSS, pp. 47-56, 2004. 